\title{
COMPORTAMENTO DE RISCO NA ADOLESCÊNCIA: UMA REVISÃO SISTEMÁTICA DA LITERATURA NACIONAL
}

\author{
CAROLINE DE FÁTIMA FRANCISCO* \\ CARLOS HENRIQUE FERREIRA DA SILVA** \\ MATHEUS HENRIQUE DA SILVA ROCHA*** \\ RENAN DE MORAIS AFONSO**** \\ LETÍCIA LOVATO DELLAZZANA-ZANON*****
}

\begin{abstract}
RESUMO
O presente trabalho tem por objetivo identificar e analisar os estudos empíricos brasileiros publicados entre os anos de 2007 e 2017 sobre comportamentos de risco de adolescentes por meio de uma revisão sistemática da literatura nacional, a fim de responder a seguinte pergunta: quais os principais comportamentos de risco de adolescentes do Brasil relatados nos estudos nacionais sobre essa temática? A busca foi realizada na base eletrônica de dados do Portal Periódicos da CAPES e teve como palavra-chave: comportamento de risco.

A busca resultou em 55 artigos e, após a aplicação dos critérios de inclusão e exclusão, 20 artigos foram analisados na íntegra. Os resultados indicaram que os comportamentos de risco mais investigados nas pesquisas nacionais no período investigado estão
\end{abstract}

* Graduanda em Psicologia, Pontifícia Universidade Católica de Campinas (PUC-Campinas), Centro de Ciências da Vida, Faculdade de Psicologia.

** Graduado em Filosofia e graduando em Psicologia, Pontifícia Universidade Católica de Campinas (PUC-Campinas), Centro de Ciências da Vida, Faculdade de Psicologia.

*** Graduando em Psicologia, Pontifícia Universidade Católica de Campinas (PUC-Campinas), Centro de Ciências da Vida, Faculdade de Psicologia.

**** Mestrando da Pontifícia Universidade Católica de Campinas (PUC-Campinas), Centro de Ciências da Vida, Programa de Pós-graduação Stricto Sensu em Psicologia.

***** Professora Pesquisadorada Pontifícia Universidade Católica de Campinas (PUC-Campinas), Centro de Ciências da Vida, Programa de Pós-graduação Stricto Sensu em Psicologia. 
relacionados com o uso de álcool e drogas e comportamento alimentar pouco saudável. Entre os profissionais que mais publicaram sobre o assunto destacam-se médicos, nutricionistas, enfermeiros e dentistas. Os resultados deste estudo chamam atenção para o fato de que comportamentos de risco de adolescentes são um tema interdisciplinar que diz respeito a diferentes áreas do conhecimento. Constata-se que pesquisas com essa população são importantes, pois seus resultados podem subsidiar políticas públicas para adolescentes e ações de prevenção, bem como orientar programas de intervenção.

Palavras-chave: comportamento de risco; adolescência; revisão sistemática.

\title{
RISK BEHAVIOR IN ADOLESCENCE: A SYSTEMATIC REVIEW OF THE NATIONAL LITERATURE
}

\begin{abstract}
The present study aimed to identify and analyze the national empirical studies published between 2007 and 2017 on adolescent risk behaviors through a systematic review of the national literature, in order to answer the following question: What are the main Brazilian adolescents risk behaviors reported in the national studies on this subject? The search was performed on the electronic database of CAPES Portal Periodicals. The keyword to the search was: risk behavior. The search resulted in 55 articles and, after applying the inclusion and exclusion criteria, 20 articles were analyzed. The results indicated that the most investigated risk behaviors in national surveys during the period investigated were alcohol and drug use and risky eating behavior. The most published professionals on the subject were doctors, nutritionists, nurses and dentists. The results of this study call attention to the fact that adolescent risk behaviors are an interdisciplinary
\end{abstract}


theme that concerns different areas of knowledge. Research done with this population is important because: its results can subsidize public policies for adolescents and prevention actions and intervention programs can be carried out from the results of these studies.

Keywords: risk behavior, adolescence, systematic review

\section{INTRODUÇÃO}

Comportamento de risco tem sido definido como qualquer conduta que possa colocar em ameaça a segurança e/ou o bem-estar da própria pessoa ou de outros indivíduos mediante um potencial de dano ou a infração às normas e regras sociais estabelecidas para evitar prejuízos (MASON et al., 2013). No que se refere ao comportamento de risco de adolescentes é consenso que esse tipo de estilo de vida se refere à participação do adolescente em atividades que possam comprometer sua saúde física e/ou mental (FEIJÓ; OLIVEIRA, 2001).

Os principais estudos de comportamentos de risco concentram-se nas seguintes áreas: consumo de álcool, comportamento sexual de risco, comportamento de risco no trânsito, comportamento antissocial, comportamento suicida, maus hábitos alimentares, dificuldades escolares e falta de atividades físicas (ZAPPE, 2014). Outros exemplos de comportamento de risco podem ser mencionados, como, por exemplo, baixos níveis de atividade física, tabagismo, consumo de drogas ilícitas e envolvimento em situações de violência, (FARIAS JUNIOR ET AL., 2009).

Resultados de uma pesquisa sobre comportamentos de risco à saúde em adolescentes no Sul do Brasil mostraram que os comportamentos mais prevalentes estão relacionados à alimentação não saudável, ao comportamento sexual de risco e ao nível insuficiente de atividade física (FARIA JÚNIOR, 2009). Chama atenção nesse estudo que mais da metade dos adolescentes da amostra estava envolvida com mais de dois comportamentos de risco simultaneamente. Esse cenário desperta cada vez mais 
preocupação entre autoridades de áreas que têm afinidade com o público em questão (DÍEZ et al., 2002), razão pela qual constata-se o crescimento da quantidade de pesquisas na temática, especialmente pelo acúmulo de vários hábitos nocivos à saúde (XING; ZHANG, 2006). Estudiosos como Paavola; Vartiainen; Haukkala (2004) à idade adulta.

Outras pesquisas sugerem que o comportamento de risco relacionado à saúde dos pais está intimamente vinculado ao desenvolvimento do mesmo comportamento nos filhos (WANG et al., 2010; EPSTEIN, BANG, BOTVIN, 2007; CAPUTO, BORDIN, 2008). Por exemplo, o uso de álcool e outras drogas pelos familiares aumentam a probabilidade de o jovem também fazer uso dessas substâncias (EPSTEIN; BANG; BOTVIN, 2007), além de aumentar a chances de o jovem desenvolver comportamento sexual de risco (CAPUTO; BORDIN, 2008). Por outro lado, estudos mostram que morar junto com os pais reduz as chances do uso de tabaco e drogas ilícitas pelos adolescentes (HORTA; HORTA; PINHEIRO, 2006). Constata-se, então, que a intimidade, a proximidade familiar, uma relação positiva com os pais e o monitoramento parental reduzem a evasão escolar e o uso de álcool (ROCHE; AHMED; BLUM, 2008), bem comoa frequência de comportamento sexual de risco (ROCHE; AHMED; BLUM, 2008; CAPALDI et al., 2002).

A convivência com pares engajados em comportamento de risco tem sido vista como fator associado ao envolvimento desse tipo de comportamento durante a adolescência (WANG et al., 2010; EPSTEIN; BANG; BOTVIN, 2007; FACUNDO; PEDRÃO, 2008). Nesse sentido, pesquisas indicam clara correlação entre o consumo de alcoól e ter amigos usuários de álcool e outras drogas (EPSTEIN; BANG; BOTVIN, 2007; FACUNDO; PEDRÃO, 2008). Assim, o fato de ter amigos e familiares usuários de drogas pode ser um fator de risco para o uso dessas substâncias durante a adolescência (KOKKEVI et al., 2007; RAPHAELLI; AZEVEDO; HALLAL, 2011). 
Quanto à faixa etária, estudos sugerem que adolescentes mais velhos, com 18 ou 19 anos de idade, são mais propensos a se engajar em comportamento de risco do que os mais novos (com 11 ou 12 anos de idade) (FLORENZANO et al., 2009; FACUNDO; PEDRÃO, 2008; FERGUS; ZIMMERMAN; CALDWELL, 2007; ZAPPE; DELL'AGLIO, 2016). O fato de os adolescentes mais velhos adotarem mais comportamentos de risco pode estar relacionado com a crescente conquista de autonomia e de independência. que impulsionam uma progressiva convivência com os pares, em detrimento do convívio com o ambiente familiar (LERNER; GALAMBOS, 1998).

Além do contexto familiar, quando se considera os espaços mais frequentados por jovens e que abrigam diversidade de estilos de vida, o ambiente escolar se desponta, pois ali é bastante comum se deparar com adolescentes que vivem problemas causados por comportamento de risco. Estão associados: baixo desempenho, falta de motivação e insatisfação com a escola (WANG et al., 2010). Um dado relevante referente à escola diz respeito ao período no qual os adolescentes estudam. Resultados de um estudo (FARIAS JÚNIOR et al., 2009) indicam que adolescentes brasileiros que estudam no período noturno apresentam maior prevalência de comportamentos de risco (consumo de drogas ilícitas e bebidas alcoólicas, não utilizam preservativos regularmente nas relações sexuais, não praticam atividade física), se comparados aos alunos adolescentes diurnos.

Quanto ao comportamento de risco alimentar, Silva, Lyra e Lima (2016, p. 1182) destacam um padrão de consumo caracterizado "pela baixa ingestão de frutas, legumes e verduras e pelo consumo excessivo de bebidas e alimentos industrializados e lanches do tipo fast-food'. De acordo com esses autores, tal conduta alimentar "pode levar ao excesso de peso e à maior probabilidade de doenças crônicas não transmissíveis (DCNT), como câncer, diabetes e morbidades e/ou fatores de risco para doenças cardiovasculares (DCV)". 
Ao tratar desse assunto, especialmente no cenário contemporâneo que é marcado pela intensa circulação de informações, torna-se oportuno mencionar que hoje, no Brasil, há políticas públicas específicas para combater a obesidade e outros transtornos alimentares. A Política Nacional de Alimentação e Nutrição (PNAN), que em 2011 promoveu sua nova edição, objetivou a melhoria das condições de alimentação, nutrição e saúde para os brasileiros, de modo a promover práticas alimentares apropriadas e saudáveis. Cabe a este órgão a vigilância alimentar e nutricional, prevenção e um cuidado integral aos danos relacionados à nutrição (MINISTÉRIO DA SAÚDE, 2012).

O Instituto Brasileiro de Geografia e Estatística - IBGE (2016) realizou pesquisas com adolescentes escolares brasileiros para identificar fatores de risco e de proteção a esse grupo de indivíduos nos anos de 2009, 2013 e 2015. A pesquisa mais recente reuniu importantes dados sobre 11 diferentes áreas de comportamento de risco: (a) uso de cigarros e outros produtos com tabaco; (b) uso de álcool; (c) drogas ilícitas; (d) uso de preservativo; (e) segurança no trajeto casa-escola; (f) brigas com utilização de armas de fogo; (g) agressões cometidas por adultos; (h) violência no trânsito; (i) bullying; (j) relações sexuais de maneira forçada; (k) e percepção da autoimagem corporal (BRASIL, 2016).

Participou da investigação um total de 102.072 adolescentes escolares do $9^{\circ}$ ano, com faixa etária de 13 a 17 anos. Os resultados demonstram que: $18,4 \%$ dos adolescentes já haviam experimentado o cigarro e 55,5\% deles ingeriram bebidas alcoólicas, sendo que $21,5 \%$ dos quais se envolveram em algum episódio de embriaguez. A respeito das drogas ilícitas: 9\% já haviam experimentado; dentre esses, 17,6\% possuem amigos que usam drogas.

Outro dado é que 27,5\% dos adolescentes disseram já terem iniciado a vida sexual e dentre esses $61,2 \%$ afirmaram o uso de preservativo na primeira vez. Além disso, 14,8\% dos adolescentes disseram ter faltado à escola nos 30 dias anteriores por não se 
sentirem seguros no trajeto, enquanto $5,7 \%$ confessaram o envolvimento em brigas com armas de fogos. Por último, 18,3\% dos adolescentes se consideram gordos, enquanto $72 \%$ dos escolares se mostraram muito satisfeitos com seu corpo (BRASIL, 2016).

Com o exposto é possível compreender a importância de estudos sobre comportamento de risco de adolescentes, pois com tais características peculiares os adolescentes podem estar mais sensíveis ao engajamento em comportamentos hábitos de risco e à exposição a situações de vulnerabilidade. Por outro lado, esta é uma fase propícia para desenvolver ações que diminuam os comportamentos de risco e os impactos que estes causam nas vidas dos adolescentes. Desse modo, considerando-se que: (a) a maior parte da população adolescente está envolvida em pelo menos um ou dois comportamentos de risco e (b) caso não identificado de maneira precoce os comportamentos podem se consolidar e trazer consequências para toda a vida do sujeito, este estudo teve como objetivo realizar uma revisão da literatura nacional a fim de investigar quais são os principais comportamentos de risco de adolescentes investigados por pesquisadores brasileiros.

\section{MÉTODO}

Este estudo de revisão sistemática de literatura tem como por objetivo responder à pergunta: quais os principais comportamentos de risco de adolescentes brasileiros relatados nos estudos nacionais sobre essa temática? Para a definição do descritor desta pesquisa consultou-se o banco de dados de Terminologia Psi da Biblioteca Virtual em Saúde de Psicologia (BVS-Psi). Após a pesquisa na BVS-Psi chegou-se ao descritor "comportamento de risco". As buscas foram realizadas no Portal Periódicos da Coordenação de Aperfeiçoamento de Pessoas de Nível Superior - Brasil (CAPES) durante o mês de novembro de 2017. O portal de periódicos da CAPES é uma biblioteca virtual que reúne e disponibiliza produções científicas nacionais e internacionais às instituições de ensino e pesquisa no Brasil. 
Utilizaram-se os critérios descritos no protocolo PRISMA (GALVÃO; PENSANI; HARRAD, 2015). A busca empreendeu as seguintes etapas: no site da CAPES (www.periodicos.capes. gov.br) foi selecionado a opção "busca avançada", limitada a um período de dez anos, 2007 a 2017. Foram aplicados os seguintes filtros: (a) artigos, (b) periódicos revisados por pares, (c) publicados no período de 2007 a 2017, (d) em língua portuguesa.

Após os resultados desta busca foram aplicados os critérios de inclusão: (a) artigos online publicados na íntegra; (b) adolescente como autor do comportamento de risco; (c) se o comportamento afeta diretamente o adolescente; (d) período de adolescência definido pela Organização Pan-americana da Saúde (10 a 21 anos) e (e) artigos empíricos. Em seguida a esses procedimentos aplicaram-se os critérios de exclusão: (a) trabalhos em que os participantes de pesquisa eram crianças e adolescentes sem discriminação de idade e (b) artigos com foco a partir da visão de terceiros sobre o adolescente.

\section{RESULTADOS}

Inicialmente foram encontrados 11.230 artigos na busca realizada e após a aplicação dos filtros de pesquisa, restaram 55 trabalhos, dentre os quais apenas um era repetido. A leitura dos resumos reduziu o universo a ser investigado a 28 artigos por meio dos critérios de inclusão, que foram detalhadamente lidos e analisados. Ao serem aplicados os critérios de exclusão previamente definidos restaram 20 artigos empíricos, publicados em português e disponíveis online na íntegra, que compuseram então a amostra deste estudo, conforme mostra a figura 1.

A investigação permitiu o exame de alguns indicadores bibliométricos da amostra, considerando que tal procedimento possibilita verificar a disseminação das informações, tamanho e caracterização da produção de conhecimento de uma determinada temática (PEDRON; BUCHELE, 2016). No que se refere ao período das publicações observou-se que o ano com maior 
Figura 1 - Fluxograma da seleção dos artigos da revisão sistemática da literatura

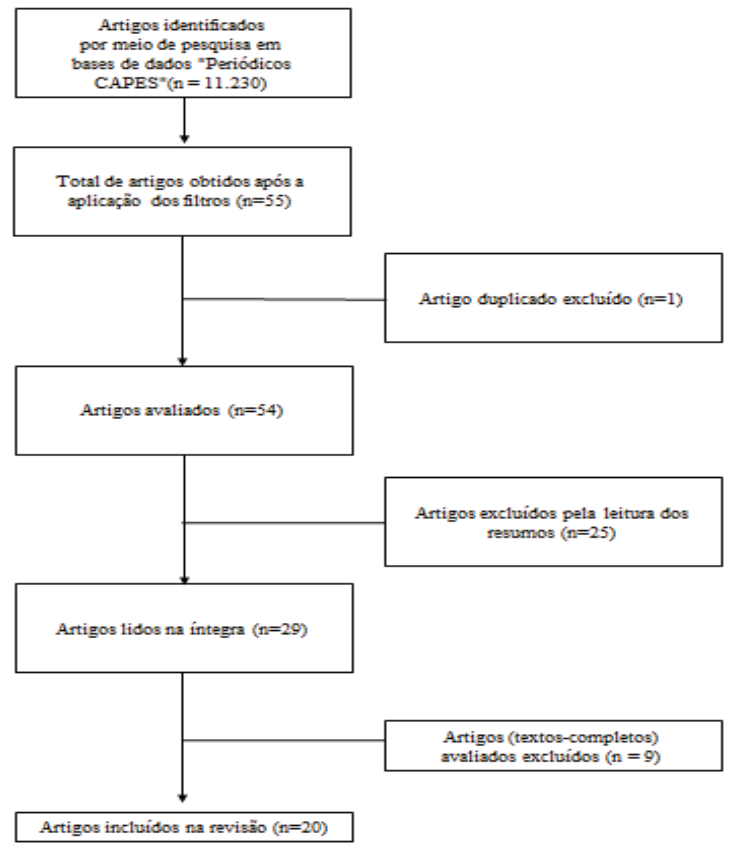

Fonte: FRANCISCO et al. (2019).

número de publicações, 2010, teve oito trabalhos, seguido de 2015 com quatro. Constatou-se ainda que: (a) em 2011, 2013 e 2017 foram publicados dois trabalhos por ano, (b) em 2009 e 2014 um trabalho anual, e (c) em 2012 e 2016 não se chegou a nenhum estudo publicado.

Dos 20 artigos analisados, seis foram produzidos por pesquisadores filiados a instituições da região Sudeste e todos têm múltipla autoria. Destacaram-se seis investigações realizadas com amostra da Pesquisa Nacional de Saúde do Escolar (PeNSE), que possui convênio com o Instituto Brasileiro de Geografia e Estatística (IBGE) e com o Ministério da Saúde. A PeNSE conta ainda com o apoio do Ministério da Educação, que pretende fornecer informações para o Sistema de Vigilância de Fatores de Risco de Doenças Crônicas não Transmissíveis (BRASIL, 2016). 
No que se refere às áreas de conhecimento dos autores dos artigos, observou-se a predominância de médicos (34 autores), seguidos por nutricionistas (15), enfermeiros (11) e dentistas (10).

O quadro 1 apresenta as principais características metodológicas dos artigos selecionados. Chamam atenção os seguintes resultados: (a) predominância de estudos quantitativos (75\% dos artigos), (b) ausência de estudos com metodologia mista, e (c) predominância do uso de questionários de autorrelato.

A região Sudeste se despontou entre as demais em vários quesitos: além de concentrar o maior número de universidades do país, são dela 32\% dos trabalhos publicados e os PPGs que mais publicaram estão na área. De suas instituições foram identificados oito PPGs, sendo quatro de Ciências da Saúde, um da Saúde Pública, um da Saúde Coletiva, um da Epidemiologia e um da Nutrição.

Tais resultados chamam atenção para a multidisciplinaridade e interdisciplinaridade da temática, tanto de profissionais, quanto de organizações interessadas. Nota-se que muitos institutos e ministérios do governo federal tiveram participação nas publicações. O envolvimento dessas instituições demonstra o interesse em estudar o comportamento de risco na adolescência e o objetivo comum de produzir conhecimento para subsidiar políticas públicas adequadas para essa população.

Visto que o principal objetivo deste trabalho foi delinear quais são os principais comportamentos de risco de adolescentes brasileiros relatados nos estudos, os resultados foram organizados de acordo com as principais temáticas presentes nos artigos selecionados: uso de álcool e drogas, comportamento alimentar, comportamento sexual de risco e prática do abortamento, violência, prática e não-prática de atividade física, bem como outros comportamentos que colocam a vida dos adolescentes em risco. 
Quadro 1 - Estudos que compuseram a revisão sistemática, classificados em função de autor, amostra, idade, instrumentos e objetivo.

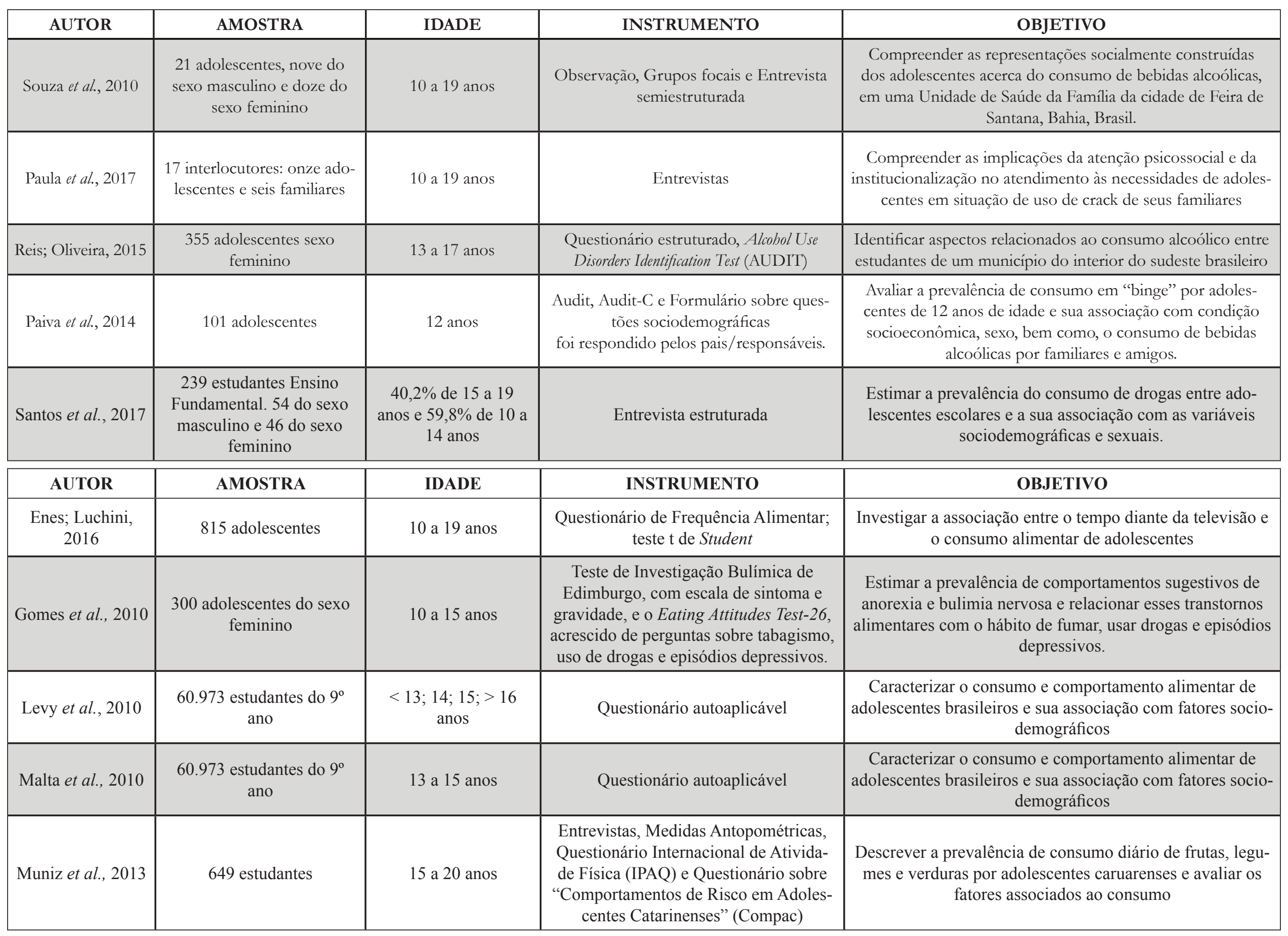




\begin{tabular}{|c|c|c|c|c|}
\hline AUTOR & AMOSTRA & IDADE & INSTRUMENTO & OBJETIVO \\
\hline Correia et al., 2011 & 2.592 adolescentes & 12 a 19 anos & Questionário semiestruturado & $\begin{array}{l}\text { Investigar as razões que levaram adolescentes a provoca- } \\
\text { rem o aborto }\end{array}$ \\
\hline Cruzeiro et al., 2010 & $\begin{array}{c}800 \text { adolescentes } 57.3 \% \text { do sexo } \\
\text { masculino e } 42.7 \% \text { do sexo } \\
\text { feminino }\end{array}$ & 15 a 18 anos & $\begin{array}{l}\text { Questionário autoaplić́vel, classificação } \\
\text { da Associação Brasileira de Institutos de } \\
\text { Pesquisa de Mercado (Abipeme) }\end{array}$ & $\begin{array}{l}\text { Avaliar o comportamento sexual de risco entre jovens de } \\
\qquad 15 \text { a } 18 \text { anos. }\end{array}$ \\
\hline Sasaki et al., 2015 & 3.099 estudantes do $9^{\circ}$ ano & 13 a 15 anos & Questionário & $\begin{array}{l}\text { Investigar a prevalência de relação sexual em adolescen- } \\
\text { tes escolares de um município brasileiro. O objetivo foi } \\
\text { investigar a prevalência de relação sexual em adolescentes } \\
\text { escolares de um município brasileiro }\end{array}$ \\
\hline Castro; Cunha; Souza, 2011 & 699 estudantes & 10 a 21 anos & Questionário auto preenchível & $\begin{array}{l}\text { Estimar a prevalência de violência entre adolescentes e } \\
\text { jovens adultos e identificar fatores associados. }\end{array}$ \\
\hline Dumith et al., 2016 & 1.112 adolescentes & 13 e 19 anos & Questionário padronizado & $\begin{array}{l}\text { Verificar a prevalência de atividade física em jovens e } \\
\text { analisar sua associação com características demográficas, } \\
\text { socioeconômicas e comportamentais. }\end{array}$ \\
\hline Hallal et al., 2010 & 60.973 estudantes do $9^{\circ}$ ano & 13 a 15 anos & $\begin{array}{l}\text { Questionários do Global School-Based } \\
\text { Student Health Survey, do Youth Riske Beha- } \\
\text { viorSurveillance System, e questionário de } \\
\text { nascimento de Pelotas, RS }\end{array}$ & $\begin{array}{l}\text { Descrever a prática de atividade física em adolescentes, } \\
\text { utilizando dados da PENSE, coletados em todas as capi- } \\
\text { tais do Brasil e no Distrito Federal em } 2009\end{array}$ \\
\hline Branco; Wagner, 2009 & 4 adolescentes & 18 anos & Estudo de caso e escala GARF & $\begin{array}{c}\text { Compreender o sucesso ou não do cumprimento da me- } \\
\text { dida de ICPAE (Interno com Possibilidade de Atividade } \\
\text { Externa) na Fase-RS (Fundação de Atendimento Socioe- } \\
\text { ducativo do Rio Grande do Sul }\end{array}$ \\
\hline Morais Neto et al., 2010 & 63.411 estudantes & 13 a 15 anos & Questionário autoaplicável & $\begin{array}{l}\text { Descrever a prevalência de fatores de risco para acidentes } \\
\text { de transporte entre adolescentes de } 13 \text { a } 15 \text { anos de esco- } \\
\text { las públicas e privadas de capitais brasileiras e do Distrito } \\
\text { Federal no ano de } 2009\end{array}$ \\
\hline Silveira et al., 2013 & $\begin{array}{l}761 \text { adolescentes. } 51.9 \% \text { sexo fe- } \\
\text { minino e } 48.1 \% \text { sexo masculino }\end{array}$ & 15 a 19 anos & $\begin{array}{c}\text { Questionário 12-Item Short-Form Health } \\
\text { Survey (SF 12). }\end{array}$ & $\begin{array}{l}\text { Investigar a qualidade de vida e sua associação com } \\
\text { fatores sociodemográficos e comportamentais entre uma } \\
\text { população de adolescentes de } 15 \text { a } 19 \text { anos }\end{array}$ \\
\hline
\end{tabular}

Fonte: FRANCISCO et al, (2019). 


\section{Uso DE ÁLCOOL E DROGAS}

Cinco artigos abordaram a temática do uso de álcool e drogas (SOUZA et al., 2010; PAULA et al., 2017; REIS; OLIVEIRA, 2015; PAIVA et al., 2015; SANTOS et al., 2017), sendo que o primeiro objetivou compreender as representações socialmente construídas por adolescentes a respeito do consumo de bebidas alcoólicas em uma Unidade de Saúde da Família da cidade de Feira de Santana-BA (SOUZA et al., 2010). Os resultados desse estudo mostram que a prática do consumo de bebida alcoólica tem caráter socializador, além de ser um ritual de passagem para os adolescentes (SOUZA et al., 2010).

O segundo (PAULA et al., 2017) estudo buscou compreender as implicações da atenção psicossocial e da institucionalização no atendimento às necessidades de 17 adolescentes, entre 10 e 19 anos, usuários de crack que utilizavam os Centros de Atenção Psicossocial (CAPS) da cidade de Fortaleza-CE. Essa pesquisa concluiu que a busca dos adolescentes usuários de crack pelo cuidado resulta, inicialmente, na institucionalização para, em seguida, incidir em encaminhados para serviços substitutivos. Constatou-se então a necessidade de fortalecimento da rede de atenção psicossocial para que o cuidado com o adolescente seja integral (PAULA et al., 2017). Já o terceiro artigo (REIS; OLIVEIRA, 2015) investigou a frequência de uso do álcool e seu consumo abusivo na vida de 638 estudantes com idade entre 13 e 17 anos, de escolas públicas da cidade de Uberlândia-MG. O estudo verificou que $80,9 \%$ dos participantes já tinham feito uso de álcool e 18,4\% faziam consumo de risco, ou seja, ingeriam grandes quantidades de bebida alcoólicas em um intervalo de tempo curto e/ou ficavam em situações vulneráveis (REIS; OLIVEIRA, 2015).

O quarto estudo (PAIVA et al., 2015) foi feito na cidade de Diamantina-MG, com 101 estudantes de 12 anos matriculados em duas escolas públicas e uma particular. O objetivo foi investigar o consumo de álcool em "binge" (ato de ingerir de cinco 
doses ou mais em uma mesma ocasião) e a relação com o sexo, a classe socioeconômica do participante e o consumo de álcool por pessoas próximas ao adolescente.

Os resultados indicaram que há alto índice de consumo de álcool em "binge" entre os participantes e que a média de idade do primeiro consumo de bebida alcoólica foi de 10,75 anos. Esses resultados estiveram associados ao uso de álcool pelo melhor amigo do adolescente indicando influencia pelos pares, e às condições socioeconômicas (PAIVA et al., 2015).

Por último, o quinto artigo (SANTOS et al., 2017) buscou medir a prevalência do consumo de drogas de adolescentes (10 a 19 anos) matriculados na rede estadual de Salvador-BA e sua relação com variáveis sociodemográficas e sexuais. A pesquisa identificou a presença de prevalência de consumo de drogas entre os adolescentes e a relação com a falta de prática religiosa, baixa escolaridade, ingresso precoce no mercado de trabalho, bem como na prática sexual (SANTOS et al., 2017).

\section{Comportamento ALIMENTAR}

Assim como o coletivo de artigos sobre o uso de álcool e drogas ressalta preocupantes informações sobre os hábitos dos adolescentes, também a temática comportamento alimentar traz pontuais referências. Esse grupo é composto por quatro trabalhos (Levy et al., 2010; Muniz et al. 2013; Enes; Llucchini, 2016; Gomes et al., 2010). Observou-se que, entre os autores desses artigos, pelo menos um era nutricionista.

O primeiro artigo visou descrever as características do consumo e do comportamento alimentar de adolescentes que participaram da Pesquisa Nacional de Saúde do Escolar (PeNSE) em 2009, matriculados no $9^{\circ}$ ano do Ensino Fundamental (LEVY et al., 2010). Os resultados dessa investigação revelam que os adolescentes tiveram não apenas consumo regular de alimentação não saudável, como também inferior ao desejado de alimentos saudáveis (LEVY et al., 2010). 
O segundo (MUNIZ et al., 2013) estudo buscou descrever a frequência com que os adolescentes do município de Caruaru-PE consomem frutas, legumes e verduras somado à necessidade de avaliar os fatores relacionados ao consumo desses alimentos (MUNIZ et al., 2013). Os resultados dessa pesquisa indicaram que o consumo desses alimentos pelos adolescentes da amostra é inferior ao aconselhado para essa faixa etária (MUNIZ et al., 2013).

O terceiro artigo (ENES; LUCCHINI, 2016) objetivou compreender a relação entre o tempo de permanência dos jovens diante da televisão e o consumo alimentar desse grupo (ENES; LUCCHINI, 2016). A amostra investigada foi composta por 815 adolescentes de duas escolas públicas do município de Piracicaba-SP. Os resultados mostram que o período em excesso em frente à televisão está relacionado a hábitos alimentares inadequados e o sedentarismo, ao intervalo temporal que os adolescentes ficam em frente à televisão (ENES; LUCCHINI, 2016).

Por fim, o estudo de Gomes et al. (2010) visou identificar a prevalência de transtornos alimentares de adolescentes do sexo feminino do Ensino Fundamental, matriculadas em escolas da rede estadual de Toledo-PR. A iniciativa pretendeu ainda relacionar os transtornos alimentares com o uso de drogas, de cigarro e de episódios depressivos. Os resultados dessa pesquisa evidenciam que o grupo analisado tem maior probabilidade de desenvolver comportamentos de risco relacionados a transtornos alimentares e a episódios depressivos.

Comportamento SEXUAL de Risco E PRÁtica do ABORTAMENTO

Aprofundando mais a investigação sobre os hábitos dos adolescentes, a terceira temática dos artigos da amostra é composta por três artigos, que têm como assunto central o comportamento sexual de risco (CORREIA et al., 2011; SASAKI et al., 2015; CRUZEIRO et al., 2010). Assim, Correia et al. (2011) realizaram um estudo transversal que teve por objetivo investigar as razões que levaram adolescentes a provocarem aborto, rela- 
cionando com as variáveis idade e tipo de escola. Participaram 2.592 jovens de 12 a 19 anos em Alagoas e no Rio Grande do Norte. Os resultados revelaram que os principais motivos para a realização do aborto foram o medo da reação dos pais, especialmente em razão da pouca idade, falta de apoio do companheiro e rejeição da gravidez.

Por sua vez, o estudo transversal de Sasaki et al. (2015) teve seu foco na investigação da prevalência de relação sexual em 3.099 adolescentes de 13 a 15 anos do $9^{\circ}$ ano de Goiânia-GO e sua associação com as variáveis sociodemográficas, comportamentos de risco à saúde e percepção da imagem corporal. A amostra foi constituída com base nos dados da PeNSE de 2009 e os resultados mostram que a prevalência de relação sexual alguma vez na vida chegou a 26,5\%. Além disso, tal comportamento foi mais predominante em adolescentes do sexo masculino, com 14 anos ou mais, que na época de realização da pesquisa estudavam em escolas públicas. Outras características prevalecentes nesses adolescentes foram não morarem com os pais, relatos de experiência de substâncias lícitas e ilícitas e terem histórico com envolvimento em brigas e de agressão familiar.

Por último, o estudo de Cruzeiro et al. (2010) avaliou os fatores associados ao número de parceiros sexuais no último ano e ao uso de preservativo nas últimas três relações sexuais entre 960 adolescentes entre 15 e 18 anos de idade do município de Pelotas-RS. Investigou-se também a associação do comportamento sexual estudado com outras variáveis sociodemográficas. Os resultados indicam que o número de parceiros sexuais nos últimos 12 meses esteve associado ao sexo (masculino/feminino), à escolaridade do adolescente, ao uso de drogas ilícitas e cigarro no mês anterior e ao consumo de bebida alcoólica antes da última relação sexual. Constatou-se também que o uso ocasional de preservativo se associou ao sexo feminino e à baixa escolaridade da mãe do participante. 


\section{ViolêNCIA E BULLYING}

Ampliando o conhecimento sobre o universo investigado, a análise desta categoria temática é composta por dois artigos (MALTA et al., 2010; CASTRO; CUNHA; SOUZA, 2011). Ao apresentam os principais resultados da PeNSE do ano de 2009, Malta et al. (2010) se atentaram à vivência de violência de adolescentes matriculados no $9^{\circ}$ ano do Ensino Fundamental. Posto que a PeNSE é aplicada pelo IBGE, a coleta de dados foi realizada em todas as capitais brasileiras e no Distrito Federal, o que possibilita ampla visão ao revelar que os estudantes do sexo masculino estão mais envolvidos em vivências de violência.

Nessa mesma categoria, Castro, Cunha e Souza (2011) realizaram um estudo transversal com 699 adolescentes com faixa etária de 10 a 21 anos da cidade de Barra do Garças-MT. Os autores buscaram estimar a prevalência de violência entre jovens e adolescentes e as causas associadas. Os resultados revelaram alto predomínio de violência entre os adolescentes, principalmente no que se refere aos jovens do sexo masculino que são usuários de bebida alcoólica, drogas e presenciam o relacionamento conturbado dos pais.

\section{Prática de atividade física e sedentarismo}

O posicionamento dos jovens com as atividades físicas foi igualmente investigado e dentre os artigos selecionados dois abordam a temática da prática de esporte ou exercício físico (HALLAL et al., 2010; DUMITH et al., 2016). O estudo de Hallal et al. (2010) teve por objetivo descrever o costume de desenvolver atividade física de adolescentes, a partir dos dados da PeNSE. Compõem a amostra 60.973 adolescentes matriculados no $9^{\circ}$ ano do Ensino Fundamental, de todas as capitais brasileiras e do Distrito Federal. Os resultados indicam baixa predominância de adolescentes ativos, além de alto número de jovens com comportamento sedentário. 
Já o segundo artigo (DUMITH et al., 2016) verificou a prevalência de atividade física em adolescentes relacionada a variáveis demográficas, socioeconômicas e comportamentais. A amostra foi de 1.112 adolescentes da cidade de Caracol, Piauí (PI). O estudo constatou que a atividade física foi considerada baixa para a amostra, principalmente para as jovens do sexo feminino, adolescentes com faixas etárias mais avançadas e também entre os indivíduos com nível socioeconômico mais baixo (DUMITH et al., 2016).

\section{OUtros COMPORTAMENTOS DE RISCO}

Esta categoria é composta pelos estudos de MALTA, et al. (2010); BRANCO, WAGNER (2009); MORAIS et al. (2010) e SILVEIRA et al. (2013). O primeiro a investigação realizada por Malta et al. (2010), que buscaram descrever os principais fatores de risco e proteção à saúde de adolescentes escolares. Uma vez que a temática desse estudo abrange todas as classificações anteriores, optou-se por inserir esse artigo nesta categoria. A amostra usada é parte da Pesquisa Nacional de Saúde do Escolar (PeNSE) de 2009. Participaram da pesquisa 60.973 adolescentes de ambos dos sexos, matriculados em escolas públicas e particulares de todas as regiões do Brasil. Os resultados desse estudo indicaram elevado consumo de alimentos gordurosos, como guloseimas e refrigerantes, além de baixo consumo de frutas. No que se refere à prática de atividade física, $43 \%$ dos adolescentes foram considerados ativos fisicamente e quase $80 \%$ ficam mais de duas horas diárias em frente à televisão. A respeito do uso de álcool e drogas, apenas 6,3\% são fumantes, $8 \%$ já fizeram uso de drogas ilícitas alguma vez. Cerca de $70 \%$ dos participantes afirmaram ter experimentado álcool, enquanto $27 \%$ fazem uso frequente de bebida alcoólica.

O artigo de Branco e Wagner (2009) avaliou quatro adolescentes infratores de 18 anos que estavam cumprindo medida com possibilidade de atividade externa, ou seja, adolescentes que 
podiam fazer atividades culturas, de lazer, esportivas, etc, fora da unidade de internação. A avaliação teve por objetivo a reinserção do adolescente na sociedade. Os resultados indicam que a intervenção realizada pelos órgãos específicos para a inclusão do jovem é deficitária, visto que após a medida a rede social dos adolescentes teve piora significativa.

Morais et al. (2010) utilizaram os dados da PeNSE de 2009 para verificar fatores de risco para acidentes de transporte terrestre. A pesquisa avaliou comportamentos de risco para acidentes como: a inobservância da obrigatoriedade do uso de cinto de segurança e de capacetes e condução de veículo por pessoa alcoolizada. Os resultados confirmam as altas taxas de mortes de jovens no Brasil causadas por acidentes de trânsito.

Finalizando a macrodivisão, a pesquisa de Silveira et al. (2013) avaliou a qualidade de vida e sua relação com fatores sociodemográficos e comportamentais. A amostra foi composta por 754 adolescentes de 15 a 19 anos da cidade de Montes Claros-MG. Os resultados indicam bons níveis de qualidade de vida dos adolescentes, visto que $45 \%$ deles praticam atividade física com frequência, não exercem atividade laboral e não consomem tabaco e álcool.

\section{DISCUSSÃO}

O objetivo deste estudo foi investigar quais são os principais comportamentos de risco de adolescentes relatados em pesquisas sobre essa temática por meio de uma revisão sistemática da literatura nacional. Os resultados indicam que os comportamentos de risco mais investigados nas pesquisas nacionais no período de 2007 a 2017 foram o uso de álcool e drogas e o comportamento alimentar. Constatou-se que o consumo de substâncias psicoativas por adolescentes é um comportamento de risco em crescimento nos últimos anos, o que pode acarretar vários prejuízos na vida desses jovens (JESUS et al., 2011). 
Além disso, pode-se inferir que o uso de álcool e tabaco foram os comportamentos de risco mais investigados pelos pesquisadores interessados na temática por se tratar de drogas lícitas e, portanto, serem de fácil acesso para qualquer adolescente. Outra razão para que o uso do álcool e do tabaco tenha sido mais estudado pode ser o fato de que a sua prática/adesão seja amplamente aceita e até mesmo incentivada em muitas famílias, o que pode ser o início de um comportamento não saudável para muitos adolescentes (CÂMARA, 2005). Não por acaso, o uso de álcool e outras drogas pelos familiares pode aumentar a probabilidade de o adolescente também fazer uso dessas substâncias (EPSTEIN; BANG; BOTVIN, 2007).

No que se refere ao comportamento alimentar, o fato dessa conduta ter sido o segundo hábito mais investigado pelos pesquisadores interessados na temática chama-se atenção para o fato de que a alimentação dos adolescentes brasileiros está sendo encarada como uma questão de saúde pública. De acordo com um levantamento realizado pelo Instituto Brasileiro Geográfico e Estatística (BRASIL, 2016), 3,1\% dos escolares com idade entre 13 e 17 estão com baixo peso, enquanto 23,7\% têm sobrepeso, ao passo que 7,8 já são considerados obesos. Nesse sentido, pesquisas feitas a partir de diferentes áreas do conhecimento que investiguem como é o padrão alimentar de adolescentes brasileiros podem subsidiam novas políticas públicas que poderão gerar avanços em relação ao enfrentamento de tal problema.

Outro aspecto relacionado ao comportamento alimentar é que esse tipo de costume tem potencial para se estender à idade adulta e gerar problemas de saúde física e mental (PAAVOLA; VARTIAINEN; HAUKKALA, 2004) durante toda a vida do sujeito. Assim, estudiosos como Silva, Lyra e Lima (2016) defendem que um padrão de consumo alimentar de risco, típico da alimentação adolescente, pode levar não apenas ao excesso de peso, mas também a maior probabilidade de desenvolver doenças 
crônicas não transmissíveis como diabetes e câncer, bem como fatores de risco para doenças cardiovasculares.

Deve-se ressaltar que dos três artigos cujo objetivo foi investigar o comportamento alimentar de adolescentes, apenas um (GOMES et al., 2010) teve o foco de investigar a ocorrência de transtornos alimentares como anorexia e bulimia. Oportuno mencionar que o fato de o comportamento alimentar de risco ter sido mais investigado do que outros hábitos preocupantes típicos da adolescência, como sexo de risco e violência, chamou a atenção dos autores deste estudo.

No que se refere às áreas de conhecimento dos autores dos artigos, observou-se predominância de médicos, seguidos por nutricionistas, enfermeiros e dentistas. A diversidade de áreas de pesquisadores encontrada neste estudo sugere que há interesse compartilhado motivado pela disposição de construir um conhecimento que proporcione melhores decisões para a elaboração de políticas públicas dirigidas a adolescentes, principalmente no que se refere à saúde deles.

Quanto às regiões do país em que foram feitos os estudos, dos 20 artigos analisados seis foram realizados por pesquisadores filiados a instituições da região Sudeste - o que já era esperado, considerando-se que essa é a região em que há maior concentração de instituições de pesquisa no país. Destacam-se seis artigos realizados com amostra da Pesquisa Nacional de Saúde do Escolar (PeNSE). Por meio de um convênio com o Ministério da Educação, o Ministério da Saúde e o IBGE, a PeNSE proporcionou a comparação das três primeiras edições desta pesquisa, entre as capitais brasileiras e o Distrito Federal. Por meio dos resultados da PeNSE, pretende-se fornecer informações para o Sistema de Vigilância de Fatores de Risco de Doenças Crônicas não Transmissíveis (BRASIL, 2016).

Deve-se ressaltar que este estudo de revisão sistemática de literatura foi escrito por psicólogos movidos por investigar quais os comportamentos de risco mais investigados pesqui- 
sados entre os anos 2007 e 2017 por estudiosos interessados neste tema, independentemente da sua área de conhecimento. O fato do uso de álcool e do tabaco ser o comportamento de risco mais investigado no período em questão caminha ao lado do crescimento da literatura sobre esse assunto e, portanto, não causou surpresa. No entanto, chamou atenção o fato de que o hábito alimentar foi o segundo tipo de comportamento de risco mais investigado. Esses resultados indicam que nos últimos anos o interesse em estudar comportamento alimentar de risco entre os adolescentes está crescendo, principalmente nos estudos diretamente desenvolvidos junto a esse público.

Considerando-se o viés da psicologia, indaga-se: de que forma o olhar do psicólogo poderia contribuir em pesquisas realizadas sobre comportamentos de risco alimentar? Acredita-se que o ideal seria planejar estudos nos quais se possa identificar comportamentos alimentares de adolescentes para, a partir dos resultados encontrados, propor ações de prevenção em conjunto com outros profissionais que ajudem na prevenção desses tipos de comportamento de risco.

Um dos resultados deste estudo é que não foram encontrados artigos em revistas específicas de psicologia, o que permite inferir que esta seja uma das limitações deste trabalho. Importante afirmar que havia, sim, a expectativa de que psicólogos estivessem entre os profissionais que mais publicam sobre o assunto, mas, no entanto, os resultados indicaram que médicos, nutricionistas, enfermeiros e dentistas foram os cientistas que mais realizaram pesquisas sobre comportamentos de risco de adolescentes no período do estudo.

Pode-se pensar que, caso outras palavras-chave tivessem sido usadas, como, por exemplo, vulnerabilidades ou fatores de risco, talvez fossem catalogados mais artigos produzidos por psicólogos. De qualquer forma, os resultados deste estudo chamam atenção para o fato de que comportamentos de risco de adolescentes são um tema interdisciplinar que diz respeito 
a diferentes áreas do conhecimento. Pesquisas feitas com essa população são importantes, pois (a) seus resultados podem subsidiar políticas públicas para adolescentes e (b) ações de prevenção e programas de intervenção podem ser realizados a partir dos resultados desses estudos.

\section{REFERÊNCIAS}

BRANCO, B. de M.; WAGNER, A. Os adolescentes infratores e o empobrecimento da rede social quando do retorno à comunidade. Ciência \& Saúde Coletiva, Rio de Janeiro, v. 14, n. 2, p. 557-566. 2009.

BRASIL. IBGE. Pesquisa Nacional de saúde do Escolar, 2016. Disponível em: <https://biblioteca.ibge.gov.br/visualizacao/livros/liv97870.pdf>. Acesso em: 17 nov. 2018.

CÂMARA, S. Comportamentos de risco entre jovens. Psico. Porto Alegre, v. 36, n. 1, p. 89-97, 2005.

CAPALDI, D. M.; STOOLMILlER, M.; CLARK, S.; OWEN, L. D. Heterosexual risk behaviors in at-risk young men from early adolescence to young adulthood: prevalence, prediction, and association with STD Contraction. Developmental Psychology, v. 38, n. 3, p. 394-406, 2002.

CAPUTO, V. G.; BORDIN, I. A. Gravidez na adolescência e uso freqüente de álcool e drogas no contexto familiar. Revista de Saúde Pública, São Paulo, v. 42 , n. 3, p. 402-410, June 2008

CASTRO, M. de L.; CUNHA, S. S. da; SOUZA, D. P O. de. Comportamento de violência e fatores associados entre estudantes de Barra do Garças, MT. Revista de Saúde Pública, São Paulo, v. 45, n. 6, p. 1054-1061. 2011.

CIAIRANO, S.; KLIEWER, W.; RABAGLIETTI, E. Adolescent Risk Behavior in Italy and The Netherlands. European Psychologist, Torino. v. 14, n. 3, p. 180-192, 2009.

CORreiA, D. S.; CAVAlCANTE, J. C.; EGITO, E. S. T.; MAIA, E. M. C. Prática do abortamento entre adolescentes: um estudo em dez escolas de Maceió (AL, Brasil). Ciência \& Saúde Coletiva. Rio de Janeiro, v. 16, n. 5, p. 2469-2476. 2011. 
CURRIE, C.; ROBERTS, C.; MORGAN, A.; SMITH, R.; SETTERTOBULTE, W.; SAMDAL, O.; RASMUSSEN, V. B. Young people's health in context: Health Behaviour in School-Aged Children (HBSC) study: international report from the 2001/2002 survey. Copenhagen: WHO Regional Office for Europe; 2004.

CRUZEIRO, A. L. S.; SOUZA, L. D. M.; SILVA, R. A.; PINHEIRO, R. T.; ROCHA, C. L. A; HORTA, B. L. Comportamento sexual de risco: fatores associados ao número de parceiros sexuais e ao uso de preservativo em adolescentes. Ciência \& Saúde Coletiva. Rio de Janeiro v. 15 (suppl 1), p. 1149-1158, 2010.

DÍEZ, J. M. B.; PÉREZ, B. Á.; FORCADELL, P. P.; PEÑACOBA, R. M.; SABATÉ, M. N.; BORONAT, A. A. Asociación entre laagrupación (clustering) de factores de riesgo cardiovascular y elriesgo de enfermedad cardiovascular. Revista Española de Salud Pública. Madri, v.76, n. 1, p. 7-15, 2002.

DUMiTH, S. C.; SANTOS, M. N.; TEIXEIRA, L. O.; CAZEIRO, C. C.; MAZZA, S. E. I.; CESAR, J. A. Prática de atividade física entre jovens em município do semiárido no Brasil. Ciência \& Saúde Coletiva. Rio de Janeiro, v. 21, n. 4, p. 1083-1093, 2016.

ENES, C. C.; LUCCHINI, B. G. Tempo excessivo diante da televisão e sua influência sobre o consumo alimentar de adolescentes. Revista de Nutrição. Campinas, v. 29, n. 3, p. 391-399, 2016.

EPSTEIN, J. A.; BANG, H.; BOTVIN, G. J. Which psychosocial factors moderate or directly affect substance use among inner-city adolescents? Addictive Behaviors, v. 32, n. 4, p. 700-713, 2007.

FACUNDO, F. R. G.; PEDRÃO, L. J. Personal and interpersonal risk factors in the consumption of illicit drugs by marginal adolescents and young people from juvenile gangs. Revista Latino-Americana de Enfermagem. Ribeirão Preto, v. 16, n. 3, p. 368-374, 2008.

FARIAS JÚNIOR, J. C.; NAHAS, M. V.; BARROS, M. V. G.; LOCH, M. R.; OLIVEIRA, E. S. A.; DE BEM, M. F. L.; LOPES, A. S. Comportamentos de risco à saúde em adolescentes no Sul do Brasil: prevalência e fatores associados. Revista Panamericana de Salud Pública, v. 25, n. 4, p. 344-352, 2009.

FEIJÓ, R. B.; OLIVEIRA, É. A. Comportamento de risco na adolescência. Jornal de Pediatria. Porto Alegre, v. 77, n. 2, p. 125-134, 2001. 
FERGUS, S.; ZIMMERMAN, M. A.; CALDWELL, C. H. Growth trajectories of sexual risk behavior in adolescence and young adulthood. American Journal of Public Health. Washington, v. 97, n. 6, p. 1096-101, 2007.

FLORENZANO U, R.; CÁCERES C, E.; VALDÉS C, M.; CALDERÓN S, S.; SANTANDER R. S.; CASASSUS T, M. Conductas de riesgo, síntomasdepresivos, auto y heteroagresiónen una muestra de adolescentes escolarizados enlaRegión Metropolitana de Santiago de Chile, 2007. Revista Chilena de Neuro-Psiquiatría. Santiago, v. 47, n. 1, p. 24-33, 2009.

GALVÃO, T. F.; PENSANI, T. S. A.; HARRAD, D. Principais itens para relatar Revisões sistemcas e Meta-análises: A recomendação PRISMA. Epidemiologia e Serviços de Saúde. Brasília, v. 24, n. 2, p. 335-342, 2015. doi: 10.5123/ S1679-49742015000200017

GOMES, J. P.; LEGNANI, E.; LEGNANI, R. F. S.; GREGÓRIO, N. P.; SOUZA, R. K. Associação entre comportamento alimentar, consumo de cigarro, drogas e episódios depressivos em adolescentes. Revista de Nutrição. Campinas, v. 23, n. 5, p. 755-762, 2010.

HALLAL, P. C.; KNUTH, A. G.; CRUZ, D. K. A.; MENDES, M. I.; MALTA, D. C. Prática de atividade física em adolescentes brasileiros. Ciência \& Saúde Coletiva. Rio de Janeiro, v. 15(suppl 2), p 3035-3042, 2010.

HORTA, R. L.; HORTA, B. L.; PINHEIRO, R. T. Drogas: famílias que protegem e que expõem adolescentes ao risco. Jornal Brasileiro de Psiquiatria. Rio de Janeiro, v. 55, n. 4, p. 268-272, 2006.

JESUS, F. B.; LIMA, F. C. A.; MARTINS, C. B. G.; MATOS, K. F.; SOUZA, S. P. S. Vulnerabilidade na adolescência: a experiência e expressão do adolescente. Revista Gaúcha de Enfermagem. Porto Alegre, v. 32, n. 2, p.359-367, 2011.

KANN, L.; MCMANUS, T.; HARRIS, W. A.; SHANKLIN, S. L.; FLINT, K. H.; QUEEN, B.; ETHIER, K. A. Youth Risk Behavior Surveillance United States, 2017. MMWR. Surveillance Summaries, Atlanta, v. 67, n. 8, p. 1-114, 2018.

KOKKEVI, A.; RICHARDSON, C.; FLORESCU, S.; KUZMAN, M.; STERGAR, E. Psychosocial correlates of substance use in adolescence: A crossnational study in six European countries. Drug and Alcohol Dependence, v. 86 , n. 1, p 67-74, 2007.

LERNER, R. M.; GALAMBOS, N. L. Adolescent development: Challenges and opportunities for research, programs, and policies. Annual Reviews Psychology, v. 49, p. 413-446, 1998. 
LEVY, R. B.; CASTRO, I. R. R.; CARDOSO, L. O.; TAVARES, L. F.; SARDINHA, L. M. V.; GOMES, F. S.; COSTA, A. W. N. Consumo e comportamento alimentar entre adolescentes brasileiros: Pesquisa Nacional de Saúde do Escolar (PeNSE), 2009. Ciência \& Saúde Coletiva. Rio de Janeiro, v.15(suppl 2), p. 3085-3097, 2010.

MALTA, D. C.; SARDINHA, L. M. V.; MENDES, I.; BARRETO, S. M.; GIATTI, L.; CASTRO, I. R. R.; CRESPO, C. Prevalência de fatores de risco e proteção de doenças crônicas não transmissíveis em adolescentes: resultados da Pesquisa Nacional de Saúde do Escolar (PeNSE), Brasil, 2009. Ciência \& Saúde Coletiva. Rio de Janeiro, v. 15(suppl 2), p. 3009-3019, 2010.

MALTA, D. C.; SOUZA, E. R.; SILVA, M. M. A.; SILVA, C. S.; ANDREAZZI, M. A. R.; CRESPO, C.; PENNA, G. O. Vivência de violência entre escolares brasileiros: resultados da Pesquisa Nacional de Saúde do Escolar (PeNSE). Ciência \& Saúde Coletiva. Rio de Janeiro, v.15(suppl 2), p. 3053-3063, 2010.

MASON, M. J.; TANNER, J. F.; PIACENTINI, M.; FREEMAN, D.; ANASTASIA, T.; BATAT, W.; ... YANG, Z. Advancing a participatory approach for youth risk behavior: Foundations, distinctions, and research directions. Journal of Business Research, v. 66, n. 8, p. 1235-1241, 2013.

MASLOWSKY, J.; BUVINGER, E.; KEATING, D. P.; STEINBERG, L.; CAUFFMAN, E. Cost-Benefit Analysis Mediation of the Relationship Between Sensation Seeking and Risk Behavior. Personality and Individual Differences, v. 51, n. 7, p. 802-806, 2011.

MINISTÉRIO DA SAÚDE. Política Nacional de Alimentação e Nutrição, 2012. Disponível em: <http://189.28.128.100/dab/docs/portaldab/publicacoes/pnan2011.pdf>. Acesso em: 20 jun. 2019.

MORAIS NETO, O. L.; MALTA, D. C.; MASCARENHAS, M. D. M.; DUARTE, E. C.; SILVA, M. M. A.; OLIVEIRA, K. B.; ... PORTO, D. L. Fatores de risco para acidentes de transporte terrestre entre adolescentes no Brasil: Pesquisa Nacional de Saúde do Escolar (PeNSE). Ciência \& Saúde Coletiva. Rio de Janeiro, v. 15(suppl 2), p. 3043-3052, 2010.

MUNIZ, L. C.; ZANINI, R. V.; SCHNEIDER, B. C.; TASSITANO, R. M.; FEITOSA, W. M. N.; GONZÁLEZ-CHICA, D. A. Prevalência e fatores associados ao consumo de frutas, legumes e verduras entre adolescentes de escolas públicas de Caruaru, PE. Ciência \& Saúde Coletiva. Rio de Janeiro, v. 18, n. 2, p. 393-404, 2013. 
PAAVOLA, M.; VARTIAINEN, E.; HAUKKALA, A. Smoking, alcohol use, and physical activity: a 13-year longitudinal study ranging from adolescence into adulthood. The Journal of Adolescent Health: Official Publication of the Society for Adolescent Medicine, v. 35, n. 3, p. 238-44, 2004.

PAIVA, P. C. P.; PAIVA, H. N.; LAMOUNIER, J. A.; FERREIRA, E. F. E; CÉSAR, C. A. S.; ZARZAR, P. M. Consumo de álcool em binge por adolescentes escolares de 12 anos de idade e sua associação com sexo, condição socioeconômica e consumo de álcool por melhores amigos e familiares. Ciência \& Saúde Coletiva. Rio de Janeiro, v. 20, n. 11, p. 3427-3435, 2015.

PAULA, M. L.; JORGE, M. S. B.; LIMA, L. L.; BEZERRA, I. C. Experiências de adolescentes em uso de crack e seus familiares com a atenção psicossocial e institucionalização. Ciência \& Saúde Coletiva. Rio de Janeiro, v. 22, n. 8, p. 2735-2744, 2017.

RAPHAELLI, C. DE O.; AZEVEDO, M. R.; HALLAL, P. C. Associação entre comportamentos de risco à saúde de pais e adolescentes em escolares de zona rural de um município do Sul do Brasil. Cadernos de Saúde Pública. Rio de Janeiro, v. 27, n. 12, p. 2429-2440, 2011.

REIS, T. G.; OLIVEIRA, L. C. M. Padrão de consumo de álcool e fatores associados entre adolescentes estudantes de escolas públicas em município do interior brasileiro. Revista Brasileira de Epidemiologia. São Paulo, v. 18, n. 1, p. 13-24, 2015.

ROCHE, K. M.; AHMED, S.; BLUM, R. W. Enduring consequences of parenting for risk behaviors from adolescence into early adulthood. Social Science \& Medicine, v. 66, n. 9, p. 2023-2034, 2008.

SANTOS, M. M.; MOTA, R. S.; CARVALHO, M. R. S.; ARAÚJO, G. S.; GOMES, N. P.; OLIVEIRA, J. F. Consumo de drogas e fatores associados: estudo transversal com adolescentes escolares do ensino fundamental. Online Brazilian Journal of Nursing. Bahia, v. 16, n. 1, p. 64, 2017.

SASAKI, R. S. A.; LELES, C. R.; MALTA, D. C.; SARDINHA, L. M. V.; FREIRE, M. C. M. Prevalência de relação sexual e fatores associados em adolescentes escolares de Goiânia, Goiás, Brasil. Ciência \& Saúde Coletiva. Rio de Janeiro, v. 20, n. 1, p. 95-104, 2015.

SILVA, D. F. O.; LYRA, C. O.; LIMA, S. C. V. C. Padrões alimentares de adolescentes e associação com fatores de risco cardiovascular: uma revisão sistemática. Ciência \& Saúde Coletiva. Rio de Janeiro, v. 21, n. 4, p. 1181-1196, 2016. 
SILVEIRA, M. F.; ALMEIDA, J. C.; FREIRE, R. S.; FERREIRA, R. C.; MARTINS, A. E. B. L.; MARCOPITO, L. F. Qualidade de vida entre adolescentes: estudo seccional empregando o SF-12. Ciência \& Saúde Coletiva. Rio de Janeiro, v. 18, n. 7, p. 2007-2015, 2013.

SOUZA, S. L.; FERRIANI, M. G. C.; SILVA, M. A. I.; GOMES, R.; SOUZA, T. C. A representação do consumo de bebidas alcoólicas para adolescentes atendidos em uma Unidade de Saúde da Família. Ciência \& Saúde Coletiva. Rio de Janeiro, v. 15, n. 3, p. 733-741, 2010.

WANG, R.-H.; HSU, H.-Y.; LIN, S.-Y.; CHENG, C.-P.; LEE, S.-L. Risk behaviours among early adolescents: risk and protective factors. Journal of Advanced Nursing, v. 66, n. 2, p. 313-323, 2010.

XING, Y.; JI, C.; ZHANG, L. Relationship of Binge Drinking and Other Health-Compromising Behaviors among Urban Adolescents in China. Journal of Adolescent Health. v. 39, n. 4, p. 495-500, 2006.

ZAPPE, J. G. Comportamento de risco na adolescência: aspectos pessoais e contextuais. 199 p. Tese (Doutorado em Psicologia) - Universidade Federal do Rio Grande do Sul, Porto Alegre, 2014.

ZAPPE, J. G; DELL'AGLIO, D. D. Risco e proteção no desenvolvimento de adolescentes que vivem em diferentes contextos: família e institucionalização. Revista Colombiana de Psicología. Bogotá, v. 25, n. 2., 2016.

\section{OS AUTORES:}

\section{CAROLINE DE FÁTIMA FRANCISCO.}

Graduanda do $9^{\circ}$ período de Psicologia pela Pontifícia Universidade Católica de Campinas (PUC-Campinas), Centro de Ciências da Vida. Bolsista de Iniciação Científica, modalidade FAPIC/Reitoria no período de 2017-2018 no Programa de Pós-graduação da Universidade. E-mail: carolineffrancisco@gmail.com

\section{CARLOS HENRIQUE FERREIRA DA SILVA.}

Graduando do $9^{\circ}$ período de Psicologia pela Pontifícia Universidade Católica de Campinas (PUC-Campinas), Centro de Ciências da Vida. Graduado em Filosofia pela Pontifícia Universidade Católica de Campinas (PUC-Campinas), modalidade Licenciatura (2014). Membro do grupo de pesquisa Psicologia da Saúde e Desenvolvimento da Criança e do Adolescente (PUC-Campinas). E-mail: chfsilva10@hotmail.com 


\section{MATHEUS HENRIQUE DA SILVA ROCHA.}

Graduando do $9^{\circ}$ período de Psicologia pela Pontifícia Universidade Católica de Campinas (PUC-Campinas), Centro de Ciências da Vida. Bolsista de Iniciação Científica, modalidade FAPIC/Reitoria no período de 2018-2019 no Programa de Pós-graduação da Universidade. Membro do grupo de pesquisa Psicologia da Saúde e Desenvolvimento da Criança e do Adolescente (PUC-Campinas). E-mail: matheushrocha4@gmail.com

\section{RENAN DE MORAIS AFONSO.}

Mestrando da Pontifícia Universidade Católica de Campinas (PUC-Campinas), Centro de Ciências da Vida, Programa de Pós-graduação Stricto Sensu em Psicologia com bolsa CNPq (2018). Atualmente realiza o Treinamento em Serviço de Psicologia no Ambulatório de Substâncias Psicoativas (ASPA) do Hospital de Clínicas da Universidade Estadual de Campinas (HC-UNICAMP), na parte de psicoterapia de base comportamental na dependência química. E-mail: renan_afonso18@hotmail.com

\section{Letícia Lovato Dellazzana-Zanon.}

Professora Pesquisadora da Pontifícia Universidade Católica de Campinas (PUC-Campinas), Centro de Ciências da Vida, Programa de Pós-graduação Stricto Sensu em Psicologia desde 2015. Pesquisadora do grupo de pesquisa Psicologia da Saúde e Desenvolvimento da Criança e do Adolescente (PUC-Campinas). É membro do grupo de trabalho Avaliação em Psicologia Positiva e Criatividade da ANPEPP. E-mail: leticiadellazzana@gmail.com 